\title{
Identification key scheme to enhance network performance in wireless body area network
}

\author{
Israa Al_Barazanchi ${ }^{1}$, Haider Rasheed Abdulshaheed ${ }^{2}$, Shihab A. Shawkat ${ }^{3}$, Siti Rahayu Binti \\ Selamat $^{4}$ \\ ${ }^{1,2}$ Baghdad College of Economic Sciences University - Baghdad, Iraq \\ ${ }^{1,2,4}$ Faculty of Information and Communication Technology, Universiti Teknikal Malaysia Melaka, Hang Tuah Jaya, 76100 Durian \\ Tunggal, Melaka, Malaysia \\ ${ }^{3}$ Directorate of Education, Salah Al-Din, Iraq
}

\begin{abstract}
Article Info
Received Jan 14, 2019

\section{Keyword:}

WBAN

Network Performance

MICS

MIMIC

Packet Loss

End to End Delay

Throughput

Medical Care Monitoring

System

ABSTRACT

Wireless Body Area Network (WBAN) refers to a group of small intelligent electronic devices placed on the human body to monitor its vital signals. the efficiency of network performance in health care applications is one of the most important prerequisites for designing an effective surveillance system. However, none of the previous studies have succeeded in proposing an effective scheme that could cover the requirements for effective network performance or the development of the WBAN system capable of sending and receiving vital patient data efficiently. The aim of this paper is to enhance the performance of the network in WBAN. In addition, to verify the transfer of patient data from sensors on the body to receivers with the least value of possible packet loss, a solution is developed that can reduce the value of packet loss, end to end delay and increase the value of throughput, relying on confirmation and matching data between the sender and receiver devices. A new scheme known as the Identification Key Scheme (IKS) is proposed based on the newly available 402-405 MHz Medical Implant Communication Service (MICS). The analysis of the network performance was implemented in real WBAN dataset and used the Multi-Parameter Intelligent Monitoring for Intensive Care (MIMIC) database. MIMIC database included the data recorded from 90 Intensive Care Unit (ICU) patients. In this study, OMNET++ was used as the network simulation tool to design and evaluate the proposed scheme. The results showed that IKS was able to reduce number of packet loss and end to end delay with increased throughput. This is an indicator of the ability of IKS in providing efficient network performance between devices in the WBAN application.
\end{abstract}

\section{Corresponding Author:}

Israa Al_Barazanchi',

Computer science department,

Baghdad College of Economic Sciences University,

Baghdad, Iraq https:///rcid.org/0000-0002-6798-6295

Email: Israa44444@gmail.com

\section{Introduction}

Wireless Body Area Networks (WBAN) have emerged as a key technology for providing real-time patient health monitoring and diagnosing many life-threatening diseases. The principle adopted by health service providers is provide convenience for patients in need of health care, allowing them comfortable of movement and constant monitoring with a reduction in health care costs. The efficiency of network performance in WBAN is one of the most important prerequisites for designing an effective surveillance system. However, 
none of the previous studies have succeeded in proposing an effective scheme that could cover the requirements for effective network performance or the development of the WBAN system capable of sending and receiving vital patient data efficiently [1]. Network performance in WBAN refers to measuring the efficiency of the network in transferring patient data from the sensors devices to the receiving devices. There are several factors that depend on measuring network efficiency such as number of packet loss, end to end delay and throughput [2]. The number of packet transmission without loss is dependent on the decrease the end to end delay and increase the value of throughput. The final throughput determines the amount of data that reached the receiving device with minimum number of packet loss.

\section{Approach of network performance in WBAN}

Network performance can be defined as the measurement Quality of Service (QoS) in transmitting data from the point of transmission to the receiving point. There are several methods use performance analysis metric to measure the performance of the network. These methods vary depending on the nature and design of the network and the area in which it is used [3]. A simulation model can also be designed to test how to efficiently control the transmission of data in a network. the efficiency of network performance in health care applications is one of the most important prerequisites for designing an effective surveillance system [4]. The purpose of any study in network performance of WAN systems to verify the transfer of patient data from sensors on the body to receivers with the least value of possible packet loss, by developing a solution that can reduce the value of packet loss, End to end delay and increase the value of throughput. Recently, most systems focused on the development of solutions to address the problem of slow network and find out what is the main cause of this slow, which may be due to the transmission wave is not suitable for the carrier medium, inefficient devices, the end of the battery device, packet loss during transport or delay, etc. The network response is the main indicator for determining packet loss, End to end delay and throughput and how it affects network traffic. The throughput that represents the amount of data being sent and received in a time unit determines the amount of packet loss during transport. None of the previous studies have succeeded in proposing an effective scheme that could cover the requirements for effective network performance or the development of the WBAN system capable of sending and receiving vital patient data efficiently [5-7]. Therefore, there is a need to improve the effectiveness of network performance in addition to improve schemes and waves transfer of WBAN. Need to enhance the performance of the network in WBAN to verify the transfer of patient data from sensors on the body to receivers with the least value of possible packet loss, by developing a solution that can reduce the value of packet loss and increase the value of throughput, relying on confirmation and matching data between the sender and receiver. In this study the approach for measure the network performance is called scheme.

\section{Problem statement}

Network performance in wireless body area network is one of the most important and necessary conditions in designing an efficient monitoring system, especially devices that are associated with the transmission of vital signals like sensors. network performance lead to matching the sender's data with the recipient's data, reduce end to end delay and increase the throughput. For that purpose, WBAN needs to enhance network performance by reducing the number packet loss, end to end delay and increase the throughput by providing an efficient communication scheme in WBAN [8]. packet loss, end to end delay and low throughput leads to incomplete data access at the monitoring devices (a doctor's phone or care center), risking the lives of the patient as the dosage of drugs or procedures depend on this data, especially comatose patients [9]. packets transfer need dependable schemes to maintain the sent and received patient data, and such schemes are ordinarily based on the Compressive Sensing (CS) approach with a substantial memory of microcontrollers required. Therefore, this study addresses the problem of packet loss, end to end delay and low throughput in WBAN by proposing a new scheme based on the Medical Implant Communication Service (MICS) band utilizing smart sensors platform to improve the transfer of patient's data in emergency room. An efficient scheme for the control devices including matching the transmission of the patient data from the transmitter to the receiver with lesser packet loss is very much needed in the WBAN [10-12].

\subsection{Identification key scheme}

This study proposes a new scheme namely Identification Key Scheme (IKS) to decrease number of packet loss, end to end delay and increase the throughput in the WBAN by studying and analyzing the network 
performance and focused on packet loss, end to end delay and increase the value of throughput. The scheme also provides appropriate transfer method under efficient network performance conditions. The analysis of the network performance was implemented in real WBAN dataset and used the Multi-Parameter Intelligent Monitoring for Intensive Care (MIMIC) database. MIMIC database included the data recorded from 90 Intensive Care Unit (ICU) patients. In this research, focusing on transportation by selecting a suitable wave for the patient's environment in the intensive care room has helped us provide a solution that can be applied outside the intensive care room too, depending on specific points to strengthen the signal and then ensure that it is received. The study assumes that there are five node sensors with one collector sensor (CS). The place of sensors around the human body in the proposed design is shown in Figure 1. The proposed scheme was based on the idea of creating a table that contained key information of each sensor in terms of the type of work and the time it required to send the information. The details that are placed in the scheme table are relied upon to make sure that the patient's data is received and no packet loss.

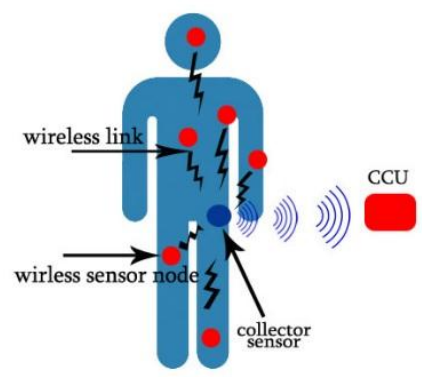

Figure 1. Location of Sensors around the Human Body with Collector Sensor

Receipt of a warning is sent to the receiver to notify them that there is a problem with the sensor or a packet loss. The programmed table is placed on a sensor that mediates the sensor set on the patient's body. This is the first stage to ensure that there is no loss of signal and then the signal was sent after it was filtered and amplified to the transmitter where there is another table that performs the same task. Patient's data is transferred from the sensors to the receiving device using efficient wave know as MICS. The MICS band offers the advantage of a miniaturized electronic device that can either be used as an implanted node or as an external node. In this thesis, a prototype sensor network is implemented by incorporating temperature and pulse rate sensors on nodes. Each developed sensor node has the capability of physiological data acquisition and local processing. The sensor node can also transmit data over the air to a remote CCU for further processing and storage. The developed system offers patient mobility as well as easy access of the patient's physiological data for the medical staff on a demand basis via the Internet. Figure 2 shows the IKS approach that includes preliminary performance data.

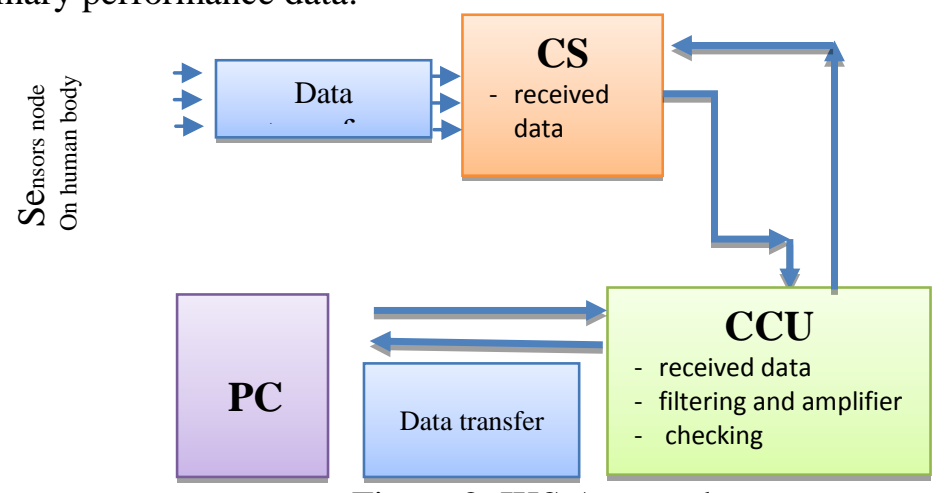

Figure 2. IKS Approach

\subsection{Structure of identification key scheme}

The input in IKS is the number of sensors; each sensor (S) has a special key that includes the type and the job of the sensor. The sensors send the signal to the collector sensor Scol and then are placed in a table called collector sensor table, then it is ascertained if all sensor data are received or not. If there is a loss, the missing data is requested from the relevant sensor based on the table showing any sensors whose signal is not received. The checking process whether there is packet loss or not depends on the parameters equation of the end-to-end delay, data rate and throughput. The main purpose of MICS band is to enhance the performance 
and reliability of data transmission in the wireless body area network by using IKS scheme. The main symbols used in the simulation are illustrated in Table 1.

\begin{tabular}{ll} 
Table 1. Summary of the Symbols used in IKS \\
Symbol & Description \\
\hline $\mathrm{M}$, & the number of sensors \\
$\mathrm{S}$ & the set of sensors \\
$\mathrm{Ch}$ & channel \\
$\mathrm{Cs}$ & Collector sensor \\
$\mathrm{Ll}$ & Link layer \\
$\mathrm{Dr}$ & Data rate \\
$\mathrm{k}$ & Packet Start Time \\
$\mathrm{Ls}$ & Link between sensor \\
$\mathrm{D}$ & Data of patient \\
$\mathrm{Ts}$ & the sending of sensor \\
$\mathrm{Mr}$ & the signals set of sensors \\
$\mathrm{EDs}$ & end to end delay of sensors \\
$\mathrm{i}$ & Direction, receiver \\
$\mathrm{Rd}$ & Receiver device \\
$\mathrm{Dr}$ & , data rate of sensors \\
$\mathrm{Ts}$, & the throughput of sensor \\
$\mathrm{L}$ & Arrival Time \\
$\mathrm{r}$ & request \\
Scol, r & the collector sensor sr \\
$\mathrm{Dloss} \mathrm{p}$ & ata loss between Sr and Scol \\
$\mathrm{Ut}$ & the Table of collector sensor \\
$\mathrm{A}$ & Message arrived \\
\hline
\end{tabular}

$M$ is the total number of sensors used in the monitoring system; $\mathrm{S}$ is considered the set of sensors; $S_{r}$ the signal that is sent from the sensor, whereby it must be $1 \leq r \leq \mathrm{M}$ that means if this condition does not verify there is packet loss, then the collector sensor reorder the data to be full in table as $\{\mathrm{m} 1, \mathrm{~m} 2, \ldots ., \mathrm{mw}\}$ with condition 1 $\leq S \leq \mathrm{M}$ to make sure the signals received are equal to the number of sensors in the collector sensor table $\mathrm{U}_{\mathrm{t}}$. The same process occurs when the data is sent from the CCU to the receiving device. The proposed scheme relies on three main stages; the first Stage data collection, the second stage representing the data in the matrix, and the third stage involving the environment data set through which the results were obtained.

\section{A. Collecting data}

It was necessary to choose an efficient method that included an organized and approved method in previous studies for the same purpose. This study adopted the Artificial Bee Colony clustering based on K-Modes (ABC-K-Modes) [8] to collect the vital data of sensors because this algorithm uses artificial method in collecting data $[9,10]$. The algorithm assumes that two sets of groups (cluster1 and cluster2) distributed sensors according to the formula of distance, which is applied randomly to any two pair sensors. Figure 3 shows the cluster algorithm (the first iteration) in the proposed enhancement. The purpose of this algorithm is to divide a number of elements ( $\mathrm{n}$ data) into a number of $\mathrm{k}$ sections in which each element is included in the section with the nearest central point. The central point represents the basis on which the data is divided, categorized, and clustered.

The result of the classification is division into groups. The algorithm's steps are as follows:

1- Assume there is n sensors ( $\mathrm{S} 1, \mathrm{~S} 2, \ldots . \mathrm{Sn})$.

2- Choose two sensors randomly (cluster 1, cluster 2).

3- Calculate the distance between the (cluster 1, cluster 2) and sensors that chosen by using distance formula: $d_{i}=\sum_{i=1}^{n}\left|C S_{i}-S_{i}\right| \quad$ for $i=1$ to $n$

4- Choose the minimum value: $\operatorname{Min}\left\{d\left(C S_{i}, S_{i}\right)\right\} \rightarrow \frac{\sum C S_{i}}{n}$

5- According to result put the sensor number in cluster. 
6- Represent the data of the two groups in the form of a matrix.

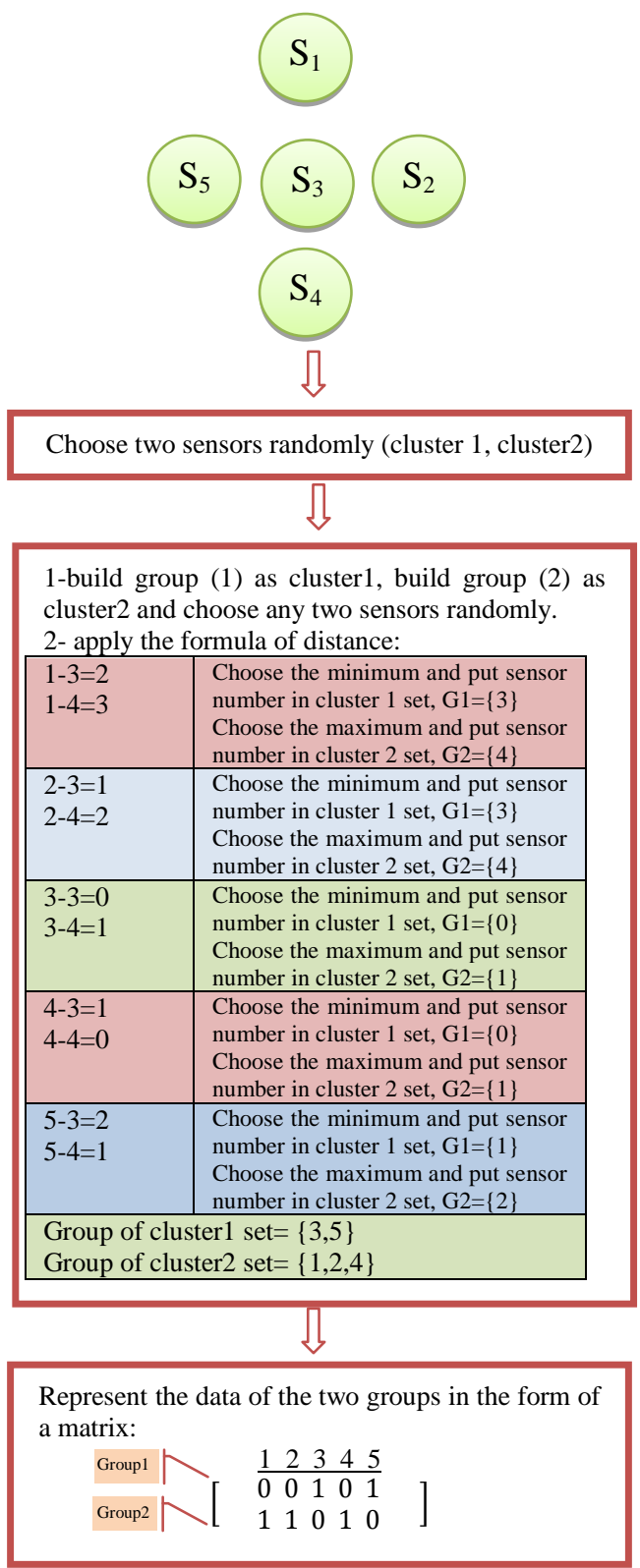

Figure 3. Cluster Algorithm (the First Iteration) in Proposed Enhancement

\section{B. Data Formulation Matrix}

Building a simulated environment requires the selection of a particular method to represent data, such as a matrix, so that it represents an environment that simulates the real world (e.g. temperature and humidity). The sensor nodes are send patients data once per time through MICS band. The time slot is represented by the symbol $t$ and the total sensors $n$. Each sensory node is required to send patient's data represented by the matrix $A(b, c)$, depending on node $b$ at time $c$, where $b=1,2 \ldots n$ and $c=1,2 \ldots t$.

- Dynamic Environment (DE): Means the mathematical environment in which the mathematical matrix will operate and is symbolized by $A$, can be defined by formula: $A=(A(b, c))_{n^{*} t}$.

Consequently, $A$ represents a matrix composed of $n$ rows and $t$ columns without packet loss because all data points in this matrix.

- Binary Matrix Formula (BMF): They represent $n \times t$ matrix, through which the value of the data can be determined in each sensor node collected in DE. BMF is denoted as F and formulated as: 


$$
F=(A(b, c))_{n * t}=\left\{\begin{array}{l}
0 \text { if } \mathrm{A}(\mathrm{b}, \mathrm{c}) \text { is packet loss } \\
1 \text { otherwise }
\end{array}\right.
$$

- Node Matrix (NM): Is a matrix for the identification of the sensor $n \times t$, recording the data that is taken from the patient's body and stored by the sensor. Based on the packet loss assumption, the element of NM is either $A(b, c)$, which were collected from the human body or zero (packet loss). For that EM consider part of NM, the symbol of EM is E and the formula below present the relation of elements:

$$
M=A \cdot F,
$$

The performance parameters are packet loss, end-to-end delay, packet loss and throughput are used to prove the efficiency of network performance in WBAN. In addition, to verify the transfer of patient data from sensors on the body to receivers with the least number of possible packet loss, end-to-end delay, and maximum throughput.

In the proposed scheme, the end-to-end delay represented as $D$, in the network are $m$ numbers of $s$ sensors. Let consider $i$ represented the direction of the sensor $s$ to the receiver device in which $S_{i}=\left\{n_{0}^{i}, n_{1}^{i}, n_{2}^{i}, \ldots \ldots . . n_{m}^{i}\right\}$, here $n_{m}^{i}$ refer to the sensor whose distance to the receiver in the direction i. the end-to-end delay for each sensor through transmission was represented as,

$$
D_{i}^{t}=\left[t_{0}, t_{1}, t_{2}, \ldots . t_{m}\right]
$$

Therefore, the mathematical formula to calculate the total end-to-end delay for each sensor data transferred from sensor to receiver device $i$ is measured by,

Where,

$$
T D_{i}=\sum L-k
$$

$T D_{i}=$ the total end-to-end delay for each sensor

$\mathrm{i}=$ receiver / direction

$\mathrm{L}=$ arrival time

$\mathrm{k}=$ packet start time

From the equation (4), measure the value of end-to-end delay $\left(T D_{i}\right)$ when $\mathrm{m}$ considered arrival time, $\mathrm{S}$ is the packet start time for nodes from human body to receiver $\mathrm{i}$. Thus, the reducing in end-to-end delay means the minimum value of end-to-end delay (MVD) for the patient's data transferred, which can be calculated by:

$$
M V D=\min \left\{T D_{i}\right\} \quad(i=1,2,3, \ldots \ldots) \ldots \ldots \ldots \ldots(5)
$$

By using the equation (5), the proposed scheme can reduce the end-to-end delay and can improve the efficiency of network performance, besides easy calculation of the total delay of all sensors by collecting the delay of each sensor on the receiving device:

$$
\text { Total delay }(\text { MVD })=(\text { delay S1 }+ \text { delay } S 2+\text { delay } S 3+\ldots)
$$

The second parameter is packet loss, generally, to calculate the number of packet loss in the proposed scheme, which can be based on the equation that calculates the value of packet loss by calculate the value of data received by reducing the data sent, as below:

$$
\text { Number of Packet loss }=\text { actual packet size }- \text { delivery packet siz }
$$

$$
P L=\left(\sum_{i=1}^{5} S_{n}\right)-\left(\sum_{i=1}^{5} Z_{i}\right)
$$

Where,

PL $=$ Number of Packet Loss

$\mathrm{S}_{\mathrm{n}}=$ Actual packet Size

$\mathrm{Z}=$ Delivery packet Size

$\mathrm{i}=$ Direction / receiver

Depending on equations (7) and (8), number of packet loss for each sensor node is measured each time data is sent and received. With the determination the number of packet loss of the missing data, the causes of packet loss and the sensor that is exposed to this problem are identified to find an efficient solution to ensure that no 
loss occurs again. At the same time, WBAN system capable of sending and receiving vital patient data efficiently. are identified in their work.

The third parameter is the throughput. This study is based on the throughput equation from the formula presented in [11]. The throughput parameter works according to the average number of data bits per second that are transmitted without packet loss. It can explain in equation 9 (Eq9):

Where

$$
\begin{gathered}
\text { Total throuput }=\frac{\text { packet deliverd }}{\text { arrival time-backet start time }} \\
T=\frac{\sum P}{\sum T-k}
\end{gathered}
$$

$\mathrm{T}=$ total throughput result

$\mathrm{P}=$ packet delivered

$\mathrm{T}=$ arrival time of patient data in receiver station

$\mathrm{K}=$ packet start time.

The decrease in the number of retransmissions in the case of packet loss can increase the throughput. Thus, increasing the number of retransmissions leads to delayed (end-to-end delay) data arrival. Throughput is at the core of the goal of health care system designers in general. It attains the measure of success of the idea developed and the efficiency of the system because it measures the impact of its success in the work environment while ensuring that the data access delay becomes less, and the protection is higher, so throughput remains the main measure of the efficiency of any WBAN system. The scheme assumes that there are a certain number of sensors, two of which are randomly selected and used as a data collector in the first instance. The data is then sent to the collector sensor and then the data is sent to CCU. The algorithm below represents the proposed scheme:

\section{ISK scheme}

Assume that: there are $M$ sensors nodes in monitoring system including collector sensor.

Initialize: give identification key 1 to $M$

\section{Input:}

M: total number of sensors;

$S:$ the set of sensors;

CS: Collector Sensor;

$b$ : sensory node;

c: time;

$A(b, c)$ : data matrix;

\section{Main process}

begin

Compute $M_{\mathrm{s}}$;

foreach $S_{\mathrm{i}} \in \mathbf{M}$ do

CALL ABC-K-MODES

if $\mathrm{CS} \in \phi$ then

foreach $S_{\mathrm{i}}$ do

$$
C S_{\mathrm{i}} \leftarrow \mathrm{CS} \mathrm{U} S_{\mathrm{i}} ;
$$

If $1 \leq S \leq M$ then

$A=(A(b, c))_{n{ }^{*} t}$

foreach $S_{\mathrm{i}}$ do

$$
\begin{aligned}
& d_{i} \leftarrow \sum_{i=1}^{M}\left|C S_{i}-S_{i}\right| \\
& \min \left\{d\left(C S_{i}, S_{i}\right)\right\} \leftarrow \frac{\sum C S_{i}}{n}
\end{aligned}
$$

$M V D=\min \left\{T D_{i}\right\}$

$T D_{i}=\sum m-k$

$T=\frac{\sum P}{\sum T-K}$

$P L=\left(\sum_{i=1}^{5} S_{n}\right)-\left(\sum_{i=1}^{5} Z_{i}\right)$

$\mathrm{PL} \leftarrow s_{n}$ 


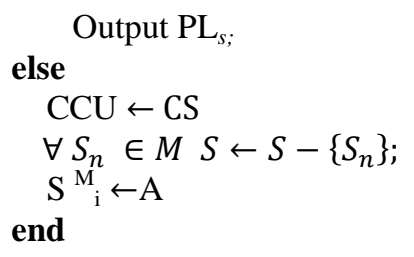

\section{Sub-algorithm ABC-K-MODES}

Input

\section{Main process}

begin

Read $m \rightarrow m=\left\{S_{1}, S_{2}, \ldots . S m\right\}$

Choose randomly two sensors $\rightarrow$ \{cluster1, cluster 2$\}$

For $(s=1 ; s \leq m ; s++)$

Find the distance $\rightarrow d_{i}=\sum_{i=1}^{m}\left|C S_{i}-S_{i}\right|$

Calculate the least result value $\left(L R V_{S}\right)$

Calculate the higher result value $\left(H R V_{S}\right)$

$$
\begin{aligned}
& \text { End for } \\
& \text { If }\left(\left(L R V_{l} \leq S_{i, 1} \leq H R V_{l}\right)\right. \\
& \text { Then } \\
& \quad \text { Class=normal } \\
& \text { Else Class=abnormal }
\end{aligned}
$$

Choose the minimum value: $\quad \operatorname{Min}\left\{d\left(C S_{i}, S_{i}\right)\right\} \rightarrow \frac{\sum C S_{i}}{m}$

If $\left(\left(L R V_{l} \leq S_{i, 1} \leq H R V_{1}\right) \& \ldots \&\left(L R V m \leq S_{i, n} \leq H R V m\right)\right)$

End if

\section{Environmental dataset}

Most of the recent studies relied on the available database for the health care records in hospitals, which are available to researchers on sites specialized in digital systems, increasing the reliability of them from $9.4 \%$ to $75.5 \%$ in the last seven years from 2008 to 2014. This study analyzes the packet loss in real WBAN dataset using the Multi-Parameter Intelligent Monitoring (MIMIC) for Intensive Care, MIMIC database includes the data recorded from 90 Intensive Care Unit (ICU) patients. Recordings of these data are a measure of the patient's vital signs periodically by clinical observation of the patient [13-16]. The database supports a variety of studies that are concerned with improving the patient's vital signaling systems. There are many reasons for choosing the dataset from MIMIC. First, the data is free and approved for all researchers around the world. Second, the data is varied and includes recordings of sensors that monitor different vital signals, and thirdly, high-precision time data [17-19]. The five vital signals were brain signal, heart signal Electro Cardio Gram (ECG), pressure, blood flow and temperature, which were used in this study of 6 patients records. The frequency of ECG signals was experimented at $500 \mathrm{HZ}$, while other sensors were experimented at $125 \mathrm{HZ}$. The data rate cycle is 1.024 second. The record was comprised of real case patients involving female and male, age $\geq 33$, with cases of heart failure, diabetes, renal failure, coma, and pneumonia. These vital signals were recorded from many ICU milieu using wire and wireless sensors. For that, these vital signals supply inclusive statues of different case.

\section{Medical Implant communication services}

In the enhancement method, a new band of Medical Implant Communication Services (MICS) related to the 402-405 MHz range with $300 \mathrm{kHz}$ channels between sensors on the human body that enable wireless communication in these medical devices to provide a high level of comfort and mobility without getting loss of patient data was used. In addition, vibrations at $402-405 \mathrm{MHz}$ have propagation characteristics that lead to the transmission of radio signals within the human body and do not pose a significant risk of interference in other radio processes in this range and miniaturization and low-power consumption. MICS band is located at an optimum frequency range that promises high-level of integration with the advanced radio frequency IC (RFIC) technology [20-23], while higher frequency causes higher penetration loss, high-level integration becomes difficult at low frequencies. In addition, there exist relatively insignificant penetration loss at these frequencies (10 dB with $10 \mathrm{~mm}$ tissue penetration). For that, the study's approval of the efficiency of MICS 
band especially when combined with the availability of the 402-405 MHZ band internationally offers an attractive frequent choice for the targeted WBSN application. After confirmation of receipt of all patient data from all sensors was sent to the CCU by MICS band, it was filtered and strengthened to be ready to send. Before being sent, it was placed in a table called the CCU table to ensure that the receipt is complete and there was no shortage of data sent, or delay in transmission was re-ordered from the collector sensor. This stage is the second point of confirmation in the proposed solution, which in turn ensures that there is no packet loss in the patient's data and therefore the receiver depends on the information sent in each period according to the nature of the work of the sensor. This data can be sent to more than one receiver with minimum packet loss. The receiver device (e.g. PC or mobile) can display all the received data on a User Display Graphic (UDG) and is also capable of storing all the data in the database system of a medical center [24-27].

\section{Simulation scenario of IKS}

OMNET++ is the program used to test scheme simulations, which aims to verify the correctness and completeness of the proposed IKS. It was followed by the simulation results, and discussions that describe the simulation conducted to evaluate the proposed scheme. The simulation plan is defined, followed by the parameter setting and performance equation that was used to get a logical table and physical table. The simulation results are then presented and discussed. There are parameters that play major roles in representing the variables on which the comparison of the proposed solution was based on previous solutions. The simulation parameters are summarized in Table 2 below:

Table 2 Simulation Parameters

\begin{tabular}{|l|l|}
\hline \multicolumn{1}{|c|}{ Parameter } & \multicolumn{1}{|c|}{ Value } \\
\hline Number of nodes & $5[15]$ \\
\hline Network area & $5 \mathrm{~m} \mathrm{[19]}$ \\
\hline Channel model & Wireless Channel /300 kHz [16] \\
\hline Transmission power & $6 \mathrm{dbm}[17]$ \\
\hline Antenna Type & Omni Antenna [18] \\
\hline Packet size & 100 bytes [19] \\
\hline Simulation time & 5000 secs [20] \\
\hline Frequencies band & MICS/402-405 MHz [21] \\
\hline Data rates & 250 kbps [22] \\
\hline $\begin{array}{l}\text { Evaluation } \\
\text { parameters }\end{array}$ & $\begin{array}{l}\text { end to end delay, Packet Loss, } \\
\text { throughput [24] }\end{array}$ \\
\hline $\begin{array}{l}\text { Considered } \\
\text { variations }\end{array}$ & Packet sizes, number of nodes [23] \\
\hline Distance (m) & $3 \mathrm{~m}[24]$ \\
\hline
\end{tabular}

\section{$5 \quad$ IKS verification and validation}

The IKS scheme was tested in a simulated environment for modeling sensor systems that transmit vital signals in the human body. The verification started by reading the first statement sent by the sensor and then reading the first data. The verification procedures were based on the techniques mentioned in the chapter three, and after the verification process the following was confirmed:

- The scheme is programmed correctly.

- The proposed scheme to reduce the value of packet loss does not contain any error or malfunction ratio.

It has been verified that reducing the packet loss has led to an increase in the percentage of data received in the receiving devices. The mechanism of ensuring that the data without a decrease in three points led to the sending of complete data and alarm in the event of any defect. To verify the efficiency and validity of the proposed improvement, the research used numerical analysis depends on the mathematical equations adopted by the proposed improvement to prove its efficiency. Data entry was repeated 30 times with different data to ensure that the model achieved the objectives set at the beginning of the work. Table 4.4 show the maximum achievable throughput average of the IKS is $7045.705 \mathrm{kbps}$ according to the packet size and comparing with (EPTS, MADM, and ESTICS) algorithms that currently used to solve the problem of packet loss in WBAN. 
Table 3. Maximum Achievable Throughput of the IKS

\begin{tabular}{|c|c|c|c|c|}
\hline Packet size (Bytes) & IKS & EPTS & MADM & ESTICS \\
\hline 10 & 8343.01 & 2545.99 & 1659.74 & 1888.16 \\
\hline 20 & 6027.16 & 4360.26 & 3213.71 & 2014.98 \\
\hline 30 & 5370.55 & 3039.87 & 3730.84 & 1333.69 \\
\hline 40 & 8442.1 & 3451.01 & 2786.23 & 2773.96 \\
\hline Average 0f Throughput & 7045.705 & 3349.2825 & 2847.63 & 2002.6975 \\
\hline
\end{tabular}

Figure 4 shows that the maximum achievable throughput of IKS goes up linearly with respect to the packet size.

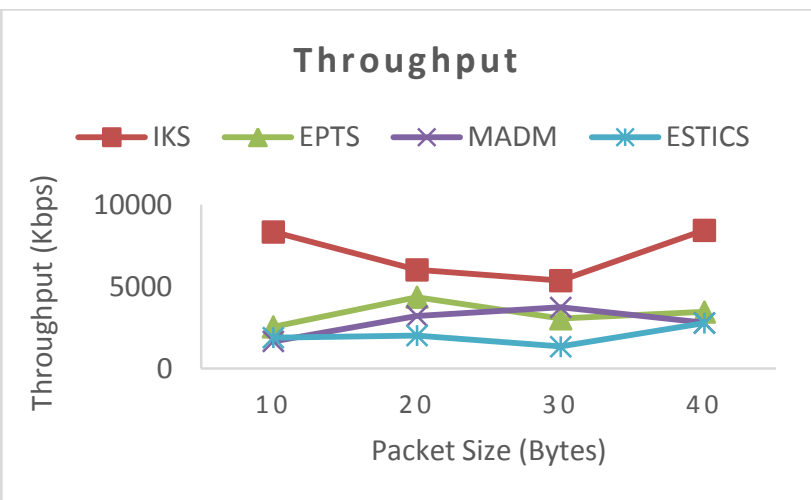

Figure 4. Maximum Achievable Throughput of IKS

Table 4 shows the end-to-end average packet delay performance for IKS in the different points of receive data. The least average of end to end is $25.325 \mathrm{kbps}$ for IKS scheme comparing with (EPTS, MADM, and ESTICS) algorithms that currently used to solve the problem of packet loss in WBAN.

Table 4. End to End Average Packet Delay Performance of IKS

\begin{tabular}{|c|c|c|c|c|}
\hline Packet size (Bytes) & IKS & EPTS & MADM & ESTICS \\
\hline 200 & 14.6 & 19.3 & 21.7 & 21.9 \\
\hline 500 & 15.9 & 18.6 & 21.4 & 21.5 \\
\hline 700 & 14.5 & 19.1 & 19.4 & 24.1 \\
\hline 1000 & 56.3 & 76.5 & 101.4 & 103.2 \\
\hline Average 0f End to End Delay & 25.325 & 33.375 & 40.975 & 42.675 \\
\hline
\end{tabular}

Figure 5. shows that the end-to-end average packet delay performance for IKS in the different points of receive data.

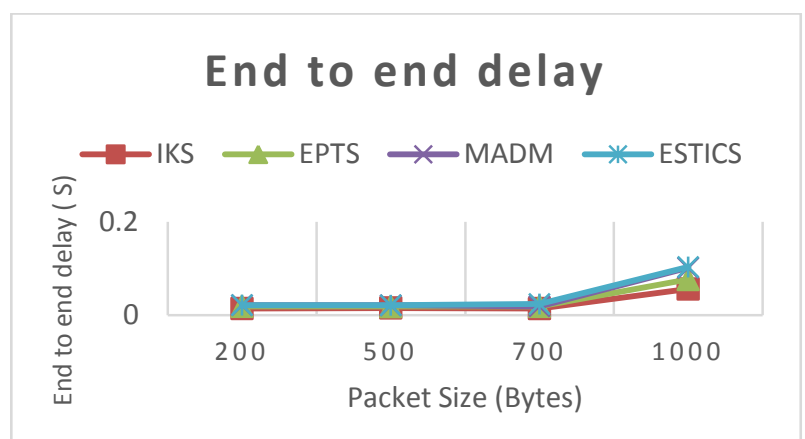

Figure 5. end-to-end average packet delay performance for IKS

Table 5 shows the result for the number of packet loss performance for IKS in the different points of receive data. The least average of end to end is $3.25 \mathrm{kbps}$ for IKS scheme comparing with (EPTS, MADM, and ESTICS) algorithms that currently used to solve the problem of packet loss in WBAN. 
Table 5. Result for Number of Packet loss Performance

\begin{tabular}{|c|c|c|c|c|}
\hline Packet size (Bytes) & IKS & EPTS & MADM & ESTICS \\
\hline 1 & 3 & 6 & 6 & 17 \\
\hline 2 & 4 & 5 & 10 & 12 \\
\hline 3 & 3 & 7 & 8 & 16 \\
\hline 4 & 3 & 8 & 9 & 15 \\
\hline Average 0f end to end delay & 3.25 & 6.5 & 8.25 & 15 \\
\hline
\end{tabular}

The curve of number packet loss increases in point one for IKS because there was delay in message transfer, but these were less than the previous algorithm used to decrease the packet loss value. Figure 6 shows the decreased packet loss value in the enhanced scheme.

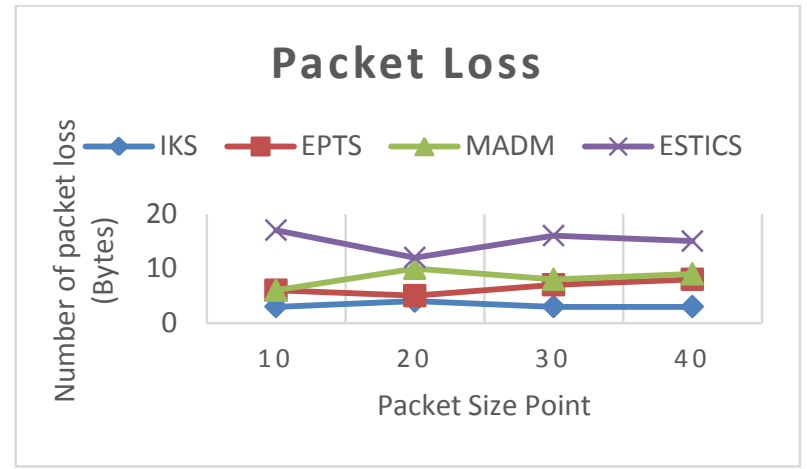

Figure 6. Number of Packet Loss in IKS Scheme.

\section{Conclusion}

The proposed scheme was based on the idea of creating a table that contained key information of each sensor in terms of the type of work and the time it required to send the information. In this research, OMNET++ was used as the network simulation tool to design and evaluate the proposed scheme. The results showed that IKS was able to reduce number of packet loss and end to end delay with increased throughput. This is an indicator of the ability of IKS in providing efficient network performance between devices in the WBAN application. Thus, this research contributed to the improvement of the efficient network performance of patient data transmission in WBAN application as well as addressing the requirement of efficient network performance by decreasing the number of packet loss. It is also anticipated that the IKS will be able to cover a larger medical environment area with additional devices that can support MICS band to connect to an ambulance or another city in the future.

\section{References}

[1] Li, X. and Ha, M., 2017. Recent Advances in Body Area Network Technology and Applications. International Journal of Wireless Information Networks, 24, pp.63-64.

[2] Ochang, P.A. and Irving, P., 2016. Performance Analysis of Wireless Network Throughput and Security Protocol Integration. International Journal of Future Generation Communication and Networking, 9 (1), pp.71-78.

[3] Farahani, G., 2017. Network Performance Enhancement with Optimization Sensor Placement in Wireless Sensor Network. International Journal of Wireless \& Mobile Networks, 9 (1), pp.09-30.

[4] Miraz, M.H., Ganie, M.A., Molvi, S.A., Ali, M., and Hussein, A.H., 2017. Simulation and Analysis of Quality of Service (QoS) Parameters of Voice over IP (VoIP) Traffic through Heterogeneous Networks. International Journal of Advanced Computer Science and Applications (IJACSA), 8 (7), pp.242-248.

[5] Liao, Y., Leeson, M., Higgins, M., and Bai, C., 2016. Analysis of In-to-Out Wireless Body Area Network Systems: Towards QoS-Aware Health Internet of Things Applications. Electronics, 5 (3), pp.38.

[6] Liao, Y., Leeson, M., Higgins, M., and Bai, C., 2016. Analysis of In-to-Out Wireless Body Area Network Systems: Towards QoS-Aware Health Internet of Things Applications. Electronics, 5 (3), pp.38.

[7] Barazanchi, I. Al, 2015. An Analysis of the Requirements for Efficient Protocols in An Analysis of the Requirements for Efficient Protocols in WBAN. Journal of Telecommunication, Electronic and Computer Engineering, 6 (July), pp.43. 
[8] Ji, J., Pang, W., Zheng, Y., Wang, Z., and Ma, Z., 2015. A Novel Artificial Bee Colony Based Clustering Algorithm for Categorical Data. PloS one, 10 (5), pp.1-17.

[9] Tri, I. and Yanto, R., 2016. A Framework of Fuzzy Partition Based on Artificial Bee Colony for Categorical Data Clustering, pp.260-263.

[10] Barazanchi, I. Al, Shibghatullah, A.S., and Selamat, S.R., 2017. A New Routing Protocols for Reducing Path Loss in Wireless Body Area Network ( WBAN ). Journal of Telecommunication, Electronic and Computer Engineering model, 9 (1), pp.1-5.

[11] Jung, B.H., Akbar, R.U., and Sung, D.K., 2012. Throughput, energy consumption, and energy efficiency of IEEE 802.15.6 body area network (BAN) MAC protocol. IEEE International Symposium on Personal, Indoor and Mobile Radio Communications, PIMRC, 5 (May 2014), pp.584-589.

[12] Johnson, A.E.W., Pollard, T.J., Shen, L., Lehman, L.W.H., Feng, M., Ghassemi, M., Moody, B., Szolovits, P., Anthony Celi, L., and Mark, R.G., 2016. MIMIC-III, a freely accessible critical care database. Scientific Data, 3, pp.1-9.

[13] Chen, M., Han, J., Fang, D., Zou, Y., and Zeng, X., 2013. An Ultra Low-Power and Area-Efficient Baseband Processor for WBAN Transmitter. Signal and Information Processing Association Annual Summit and Conference (APSIPA), 61 (20), pp.1-4.

[14] Akbar, M.S., Yu, H., and Cang, S., 2016. Delay, Reliability, and Throughput Based QoS Profile : A MAC Layer Performance Optimization Mechanism for Biomedical Applications in Wireless Body Area Sensor Networks. Journal of Sensors, 2016 (7), pp.17.

[15] Zou, Z., Bao, Y., Deng, F., and Li, H., 2015. An Approach of Reliable Data Transmission With Random Redundancy for Wireless Sensors in Structural Health Monitoring. IEEE SENSORS JOURNAL, 15 (2), pp.809-818.

[16] Yu et al., 2015. A Study on Data Loss Compensation of WiFi- Based Wireless Sensor Networks for Structural Health Monitoring. Journal, Ieee Sensors, 13597 (1).

[17] Roy, 2013. Dynamic Channel Modeling for Multi-Sensor Body Area Networks. IEEE Transactions on Antennas and Propagation, 61 (4), pp.1-9.

[18] Wei, Y. and Roblin, C., 2012. Multislot Antenna with a Screening Backplane for UWB WBAN Applications. International Journal of Antennas and Propagation, 2012.

[19] Nadeem, Q., Javaid, N., Mohammad, S.N., Khan, M.Y., Sarfraz, S., and Gull, M., 2013. SIMPLE: Stable Increased-throughput Multi-hop Protocol for Link Efficiency in Wireless Body Area Networks. Broadband and Wireless Computing, Communication and Applications (BWCCA), 2013 Eighth International Conference. IEEE (pp. 221-226).

[20] Le, T.T.T. and Moh, S., 2017. Link Scheduling Algorithm with Interference Prediction for Multiple Mobile WBANs. Sensors, 17 (10), pp.2231.

[21] Jacob, A.K. and Jacob, L., 2015. Energy Efficient MAC for QoS Traffic in Wireless Body Area Network. International Journal of Distributed Sensor Networks, 11 (2), pp.40-52.

[22] Pflug, H.W., Visser, H.J., Kiyani, N.F., Dolmans, G., Philips, K., Kanda, K., Hamaminato, M., and Masui, S., 2014. Radio Channel Characterization for $400 \mathrm{MHz}$ Implanted Devices. In Wireless Communications and Networking Conference (WCNC), 2014 . IEEE., 1 (3), pp.293-298.

[23] Le, T.T.T. and Moh, S., 2017. Link Scheduling Algorithm with Interference Prediction for Multiple Mobile WBANs. Sensors, 17 (10), pp.2231.

[24] Prameela, S., 2017. Obfuscation-based Delay-Aware Confidential Data Discovery and Dissemination Protocol in Wireless Body Area Networks. International Journal of Applied Engineering Research, 12 (18), pp.7990-7997.

[25] Zaouiat and Latif, 2017. Performances Comparison of IEEE 802.15.6 and IEEE 802.15.4. International Journal of Advanced Computer Science and Applications(IJACSA), 8 (11), pp.461-467.

[26] B. Durakovic, "Design of Experiments Application, Concepts, Examples: State of the Art," Periodicals of Engineering and Natural Scinces, vol. 5, no. 3, p. 421-439, 2017.

[27] Ali, A., Ming, Y., Chakraborty, S., and Iram, S., 2017. A Comprehensive Survey on Real-Time Applications of WSN. Future Internet, 9 (4), pp.77. 